\title{
Anti-Müllerian Hormone as a Surrogate Marker for Hormonal Dysfunction and Sonographic Pattern in Polycystic ovarian syndrome
}

\author{
Priyanka Sharma ${ }^{1}$, Raina Chawla ${ }^{2}$, Rashmi Ahuja ${ }^{3}$, Usha Gupta ${ }^{4}$
}

\begin{abstract}
Aim: To examine the association of serum anti-Müllerian hormone (AMH) levels with biochemical derangement and sonographic appearance in PCOS women and to examine its role as a possible marker for diagnosis and prognosis of polycystic ovarian syndrome (PCOS).

Materials and methods: This case-control study was conducted on patients attending the Gynecology OPD at ESIC Medical College and Hospital, Faridabad, Haryana from March 2017 to September 2017.45 PCOS women and controls between 18 years and 45 years were recruited. Relevant clinical, biochemical, and sonographic data were collected. Linear regression was used to study the association between biochemical and sonographic variables and PCOS. Receiver-operating characteristic (ROC) curve analysis was performed for AMH, LH/FSH ratio, antral follicle count (AFC), and mean ovarian volume. The AMH cut-off value was determined for the prediction of PCOS.

Results: Mean AMH levels were significantly higher in the PCOS group ( $5.9 \mathrm{v} / \mathrm{s} 2.36 \mathrm{ng} / \mathrm{mL}$ ). In PCOS women, AMH levels positively correlated with AFC, mean ovarian volume, as well as androstenedione and testosterone levels. In controls, a significant positive correlation was found with serum testosterone and mean ovarian volume. An AMH had greater diagnostic potential for detecting PCOS compared to AFC, mean ovarian volume, and LH/FSH ratio. A threshold AMH value of $3.98 \mathrm{ng} / \mathrm{mL}$ had a sensitivity of $82.2 \%$ and a specificity of $93.3 \%$ for diagnosing PCOS.

Conclusion: Serum AMH levels correlate well with hyperandrogenemia and sonographic features of PCOS and could be used as a marker for hormonal dysfunction and disease severity.

Clinical significance: An AMH is a promising marker that could improve the diagnosis of PCOS and also help in establishing disease severity. Keywords: Anti-Müllerian hormone, Hyperandrogenemia, Polycystic ovarian syndrome.

Journal of South Asian Federation of Obstetrics and Gynaecology (2019): 10.5005/jp-journals-10006-1674
\end{abstract}

\section{INTRODUCTION}

Polycystic ovarian syndrome (PCOS) affects $6.6-8 \%$ of women in the reproductive age group and is a major cause of anovulatory infertility. ${ }^{1,2}$ Androgen excess and anovulation constitute its major pathophysiology. ${ }^{3}$ Various criteria have been proposed for the diagnosis of PCOS and they all rely upon a combination of hyperandrogenism/hyperandrogenemia, anovulation, and polycystic ovarian morphology. To date, there are no objectively defined and precisely measurable markers to define and prognosticate PCOS. The Rotterdam criteria, which is the most widely accepted standard, rely on sonography as one of the cornerstones for diagnosis. However, development of high-resolution sonographic techniques, the inter and intra-observer variability in assessment of what constitutes polycystic ovarian morphology, ${ }^{4}$ the technical difficulties in performing ultrasound in young or obese females, and the variety in phenotypes have necessitated the establishment of a well-defined diagnostic marker for PCOS.

The AMH, a member of TGF- $\beta$ family, ${ }^{5}$ which is secreted by granulosa cells of small antral and preantral follicles and regulates follicular development, ${ }^{6}$ is one such marker. In PCOS, impaired folliculogenesis may result in excessive accumulation of pre-antral and small antral follicles, which may ultimately cause increased AMH levels. AMH levels correlate with ovarian follicular count ${ }^{6}$ and studies suggest that it may also be associated with ovarian hyperandrogenemia ${ }^{7,8}$ in both PCOS and healthy women. Evidence suggests that $\mathrm{AMH}$ could be utilized as an effective surrogate for ovulatory dysfunction as it correlates well with sonographic features and hormonal picture in PCOS. This study aimed to
${ }^{1-4}$ Department of Obstetrics and Gynaecology, ESIC Medical College and Hospital, Faridabad, Haryana, India

Corresponding Author: Priyanka Sharma, Department of Obstetrics and Gynaecology, ESIC Medical College and Hospital, Faridabad, Haryana, India, Phone: +91 9958984798, e-mail: priyanka_labelle@ yahoo.co.in

How to cite this article: Sharma P, Chawla R, Ahuja R, et al. AntiMüllerian Hormone as a Surrogate Marker for Hormonal Dysfunction and Sonographic Pattern in Polycystic ovarian syndrome. J South Asian Feder Obst Gynae 2019;11(3):175-180.

Source of support: Nil

Conflict of interest: None

examine the association of serum AMH levels with biochemical derangement and sonographic appearance in PCOS patients and to examine its role as a surrogate marker for diagnosis of PCOS.

\section{Materials and Methods}

This cross-sectional study was conducted at the outpatient Gynecology Clinic, Department of Obstetrics and Gynaecology, ESIC Medical College and Hospital, Faridabad, Haryana from March 2017 to September 2017. An estimated 45 women aged between 18 and 45 years attending the gynecology OPD who fulfilled the Rotterdam criteria for PCOS were enrolled for this study. Agematched controls were recruited from normo-ovulatory women attending the gynecology OPD. Informed consent was obtained from all participants. Approval was taken from the Institutional 
Ethical Committee before commencing the study. As per the Rotterdam/ESHRE criteria 2003, ${ }^{9}$ PCOS was defined as the presence of at least two of the following:

- Oligo/anovulation

- Hyperandrogenism and/or hyperandrogenemia

- Polycystic ovaries ( $>12$ follicles measuring 2-9 mm in one/both ovaries and/or increased ovarian volume $>10 \mathrm{~mL}$ in one or both ovaries)

Women with hepatic, renal and autoimmune diseases, abnormal serum albumin, overt hyper/hypothyroidism, and adrenal disorders were excluded. Those with the history of use of hormones, ovulation-induction drugs, and insulin-sensitizing drugs in the past 3 months were excluded from the study. Past history of ovarian drilling was also an exclusion criterion.

Relevant clinical data such as age, number of menstrual cycles per year, and history of acne and hirsutism were obtained. Anovulation was defined as the absence of spontaneous menses for 3 months. Oligo-ovulation was defined as fewer than 9 menstrual cycles per year. Hyperandrogenism was determined by hirsuitism (Ferriman Gallaway score $>8$ ) and/or the presence of acne. Hyperandrogenemia was defined as serum total testosterone $>2.5$ $\mathrm{nmol} / \mathrm{L}$ or free testosterone $>0.6 \mathrm{nmol} / \mathrm{L}$. Body mass index (BMI) was calculated as weight $(\mathrm{kg}) / \mathrm{height}\left(\mathrm{m}^{2}\right)$.

\section{Serum Sampling}

An estimated $8 \mathrm{~mL}$ blood was drawn between days 2 and 5 of natural or progesterone-induced menses in overnight-fasting women. Of this blood, $6 \mathrm{~mL}$ was immediately used for determination of serum FSH, LH, TSH, prolactin, androstenedione, DHEAS, total testosterone, SHBG, fasting insulin, hs-CRP, SGOT, SGPT, serum albumin, $\mathrm{LDL}, \mathrm{HDL}$, and total cholesterol levels. A total of $2 \mathrm{~mL}$ was stored in lithium heparin test tubes at $4^{\circ} \mathrm{C}$ and analyzed for serum $\mathrm{AMH}$ levels within 24 hours.

\section{AMH Assay}

Samples were brought to room temperature $\left(23 \pm 2^{\circ} \mathrm{C}\right)$. AMH levels were assessed using the Ultrasensitive AMH/MIS ELISA (AL-105i) (Ansh Labs, Webster, Texas, USA), which is a quantitative threestep sandwich-type immunoassay. The limit of detection was $0.023 \mathrm{ng} / \mathrm{mL}$ with $95 \%$ probability. The inter-assay and intra-assay coefficients of variation were $3.2 \%$ and $3.8 \%$, respectively.

\section{Ultrasound Examination}

A transvaginal scan was performed with a Philips C9-4v Endocavitary Probe between days 2 and 5 of the menstrual cycle. Where transvaginal scanning was not acceptable, a transabdominal scan was performed using a Philips C5-2 Convex Probe. Ovarian volume was calculated as $0.532 \times$ length $\times$ width $\times$ height. Antral follicle count included all follicles between 2 and $9 \mathrm{~mm}$ size. All measurements were taken on Philips ClearVue 350 (Philips Ultrasound, Inc., Bothell, WA, USA). Those with ovarian cysts or lead follicle $>10 \mathrm{~mm}$ in diameter were excluded from the study.

\section{Statistical Analysis}

Statistical analysis was performed by SPSS Version 16 (SPSS Inc, Chicago, IL, USA). Equality of means was compared using student's $t$ test. Categorical variables were analyzed by Chi-square test. A $p$ value $<0.05$ was considered significant. Linear regression was used to study the association between biochemical and sonographic variables and PCOS. Receiver-operating characteristic
(ROC) curve analysis was performed for $\mathrm{AMH}, \mathrm{LH} / \mathrm{FSH}$ ratio, antral follicle count (AFC), and mean ovarian volume. AUC estimates were calculated for each parameter to determine the probability of accurately discriminating between PCOS cases and controls. An optimal AMH cut-off value was determined for prediction of PCOS with acceptable sensitivity and specificity.

\section{Results}

The baseline clinical, hormonal, and sonographic characteristics of cases and controls are listed in Table 1. Cases and controls were similar with respect to age and BMI. Mean serum AMH levels were significantly higher in the PCOS group compared to controls $(5.9 \pm 2.0 \mathrm{ng} / \mathrm{mL}$ vs $2.36 \pm 0.88 \mathrm{ng} / \mathrm{mL}, p<0.05)$. The mean serum LH levels; LH/FSH ratio; serum-free testosterone, androstenedione, and DHEAS levels; ovarian volume; and antral follicle count were significantly higher in the PCOS group compared to controls. There was no statistically significant difference in the two groups with respect to clinical hyperandrogenism, serum FSH, TSH, Prolactin, SHBG, fasting insulin levels, and lipid profile.

In PCOS women, AMH levels correlated negatively with age $(r=-0.420)$ and BMI $(r=-0.699)$. A significant positive correlation was observed between AMH and androstenedione levels $(r=0.508)$ and serum testosterone levels $(r=0.376)$. No such correlation was observed with DHEAS. Similarly no correlation was observed between AMH levels and lipid profile or fasting insulin levels. AMH levels correlated strongly with both sonographic markers studied-AFC $(r=0.583)$ and mean ovarian volume $(r=0.591)$ (Table 2 and Fig. 1).

In controls, unlike in cases, no correlation was observed with age or BMI. AMH levels correlated positively with serum FSH levels $(r=0.351)$, testosterone levels $(r=0.592)$, and mean ovarian volume $(r=0.511)$ (Table 2 and Fig. 2).

Table 1: Clinical, biochemical, and sonographic characteristics of PCOS and controls

\begin{tabular}{lllc}
\hline & & $\begin{array}{l}\text { Controls } \\
(n=45)\end{array}$ & Significance \\
\hline Age (year) & $24.1 \pm 4.7$ & $25.2 \pm 4.6$ & 0.25 \\
BMI $\left(\mathrm{kg} / \mathrm{m}^{2}\right)$ & $25.0 \pm 5.2$ & $22.9 \pm 2.9$ & 0.18 \\
AMH $(\mathrm{ng} / \mathrm{mL})$ & $5.96 \pm 2.0$ & $2.36 \pm 0.88$ & $<0.05$ \\
Ferriman gallaway score & 13 & 10 & 0.468 \\
FSH (mlU/mL) & $7.0 \pm 3.1$ & $6.2 \pm 2.5$ & 0.19 \\
LH (mIU/mL) & $15.8 \pm 4.2$ & $6.3 \pm 2.6$ & $<0.001$ \\
LH/FSH & $2.6 \pm 1.7$ & $1.1 \pm 0.5$ & $<0.001$ \\
TSH (mIU/L) & $2.2 \pm 1.6$ & $2.0 \pm 1.4$ & 0.55 \\
Prolactin (ng/mL) & $14.8 \pm 7.2$ & $15.1 \pm 6.4$ & 0.83 \\
Testosterone (nmol/L) & $1.9 \pm 1.6$ & $0.5 \pm 0.38$ & $<0.05$ \\
Androstenedione & $2.4 \pm 0.7$ & $1.5 \pm 0.6$ & $<0.05$ \\
(nmol/L) & & & \\
DHEAS (ng/dL) & $172.9 \pm 29.3$ & $156.0 \pm 38.8$ & 0.02 \\
SHBG (nmol/L) & $44.1 \pm 17.5$ & $40.7 \pm 15.0$ & 0.34 \\
Fasting insulin (mIU/L) & $8.4 \pm 3.9$ & $7.3 \pm 2.4$ & 0.11 \\
HDL (mg/dL) & $41.0 \pm 4.4$ & $42.5 \pm 3.8$ & 0.09 \\
TG (mg/dL) & $94.9 \pm 26.2$ & $90.2 \pm 23.4$ & 0.39 \\
Total CH (mg/dL) & $151.6 \pm 15.7$ & $147 \pm 14.3$ & 0.15 \\
Ovarian volume (cm $\left.{ }^{3}\right)$ & $14.9 \pm 4.0$ & $7.5 \pm 1.8$ & $<0.001$ \\
AFC & $16.3 \pm 6.4$ & $6.2 \pm 1.9$ & $<0.001$ \\
AMH/AFC & $0.398 \pm 0.13$ & $0.412 \pm 0.19$ & 0.68 \\
\hline & & &
\end{tabular}


Table 2: Correlation of AMH with various biochemical and sonographic characteristics in PCOS cases and controls

\begin{tabular}{lccccc}
\hline & \multicolumn{2}{c}{ Cases $(n=45)$} & & \multicolumn{2}{c}{ Controls $(n=45)$} \\
\cline { 2 - 3 } \cline { 6 - 7 } & rvalue & $p$ value & & rvalue & pvalue \\
\hline Age & -0.420 & $<0.05$ & & -0.013 & 0.935 \\
BMI & -0.699 & $<0.05$ & & -0.204 & 0.179 \\
FSH & -0.278 & 0.064 & & 0.351 & $<0.05$ \\
LH & -0.207 & 0.172 & & 0.036 & 0.812 \\
LH/FSH & 0.119 & 0.436 & & -0.262 & 0.082 \\
TSH & -0.150 & 0.326 & & -0.035 & 0.821 \\
Prolactin & 0.152 & 0.320 & & 0.072 & 0.638 \\
Testosterone & 0.376 & $<0.05$ & & 0.582 & $<0.05$ \\
Androstenedione & 0.508 & $<0.05$ & & 0.042 & 0.782 \\
SHBG & -0.102 & 0.506 & & 0.005 & 0.976 \\
DHEAS & 0.117 & 0.446 & & 0.093 & 0.542 \\
Fasting insulin & -0.155 & 0.310 & & 0.176 & 0.247 \\
HDL & 0.204 & 0.179 & & 0.201 & 0.186 \\
TG & -0.45 & 0.769 & & -0.161 & 0.290 \\
Total CH & -0.182 & 0.231 & & -0.031 & 0.842 \\
Mean ovarian volume & 0.583 & $<0.05$ & & 0.511 & $<0.05$ \\
Antral follicle count & 0.591 & $<0.05$ & 0.132 & 0.386 \\
\hline
\end{tabular}
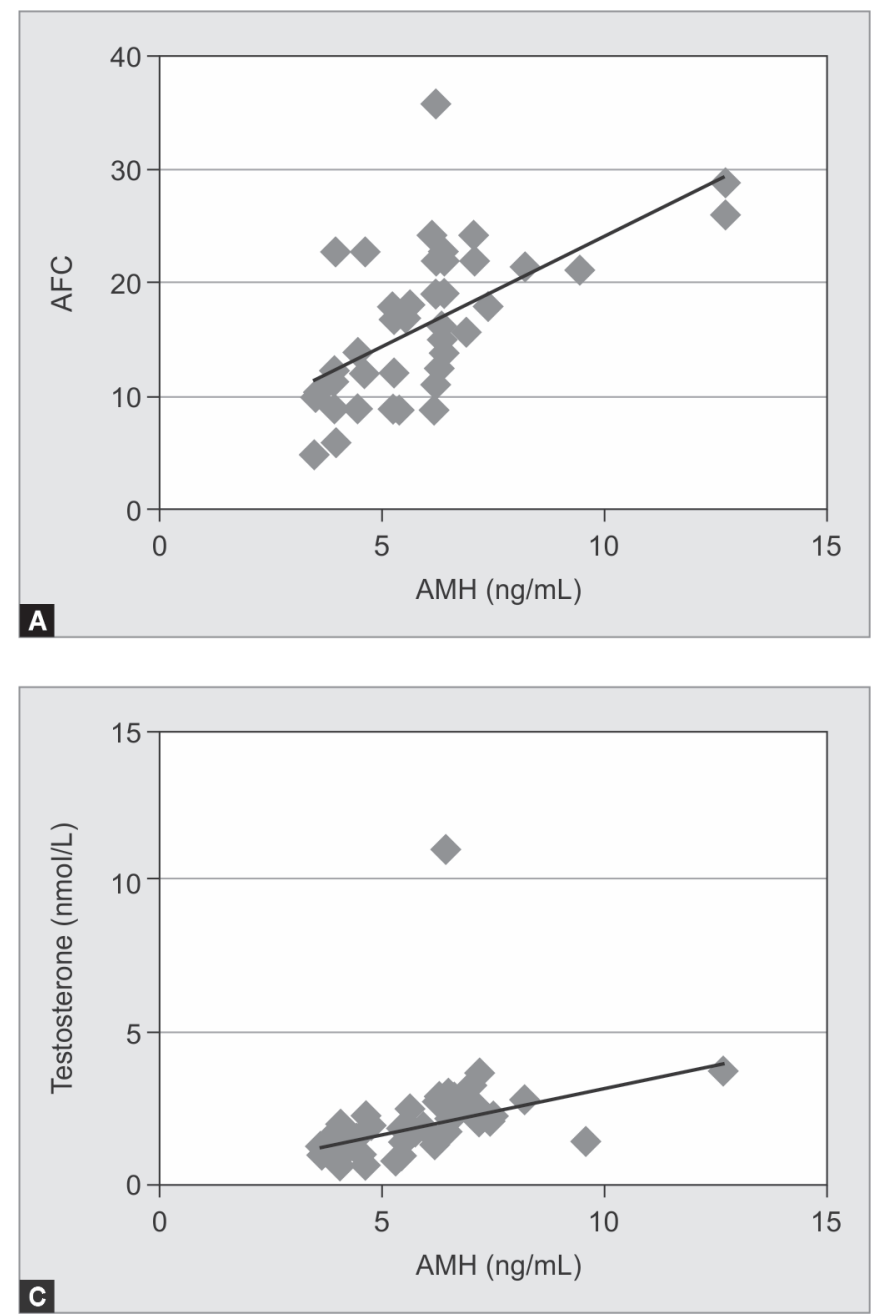

The diagnostic potential of serum $\mathrm{AMH}$ was analyzed by an ROC curve analysis. AUC for AMH was 0.987. AMH had greater diagnostic potential for detecting PCOS compared to AFC, mean ovarian volume, and LH/FSH ratio (Fig. 3).

A threshold AMH value of $3.98 \mathrm{ng} / \mathrm{mL}$ had a sensitivity of $82.2 \%$ and a specificity of $93.3 \%$ for diagnosing PCOS.

\section{Discussion}

Polycystic ovarian syndrome is a multiorgan endocrine disorder with considerable phenotypic heterogeneity and ever-changing diagnostic criteria which are subject to interobserver and intraobserver variability. There is, therefore, a need for identification of more precise and objective markers for the diagnosis and prognosis of PCOS.

Since AMH is produced by pre-antral and small antral follicles, its levels are expected to an increase in PCOS, where such follicles are in abundance. AMH likely exerts an inhibitory effect on the FSH-mediated folliculogenesis process and results in anovulation. A higher AMH/AFC ratio observed in PCOS indicates not only a greater number of antral follicles but also an increased production of AMH per antral follicle. ${ }^{10}$ This observation is supported by in vitro studies, which also demonstrate greater $\mathrm{AMH}$ production per granulosa cell in polycystic ovaries compared to controls. ${ }^{11}$ We observed significantly higher serum AMH levels in PCOS
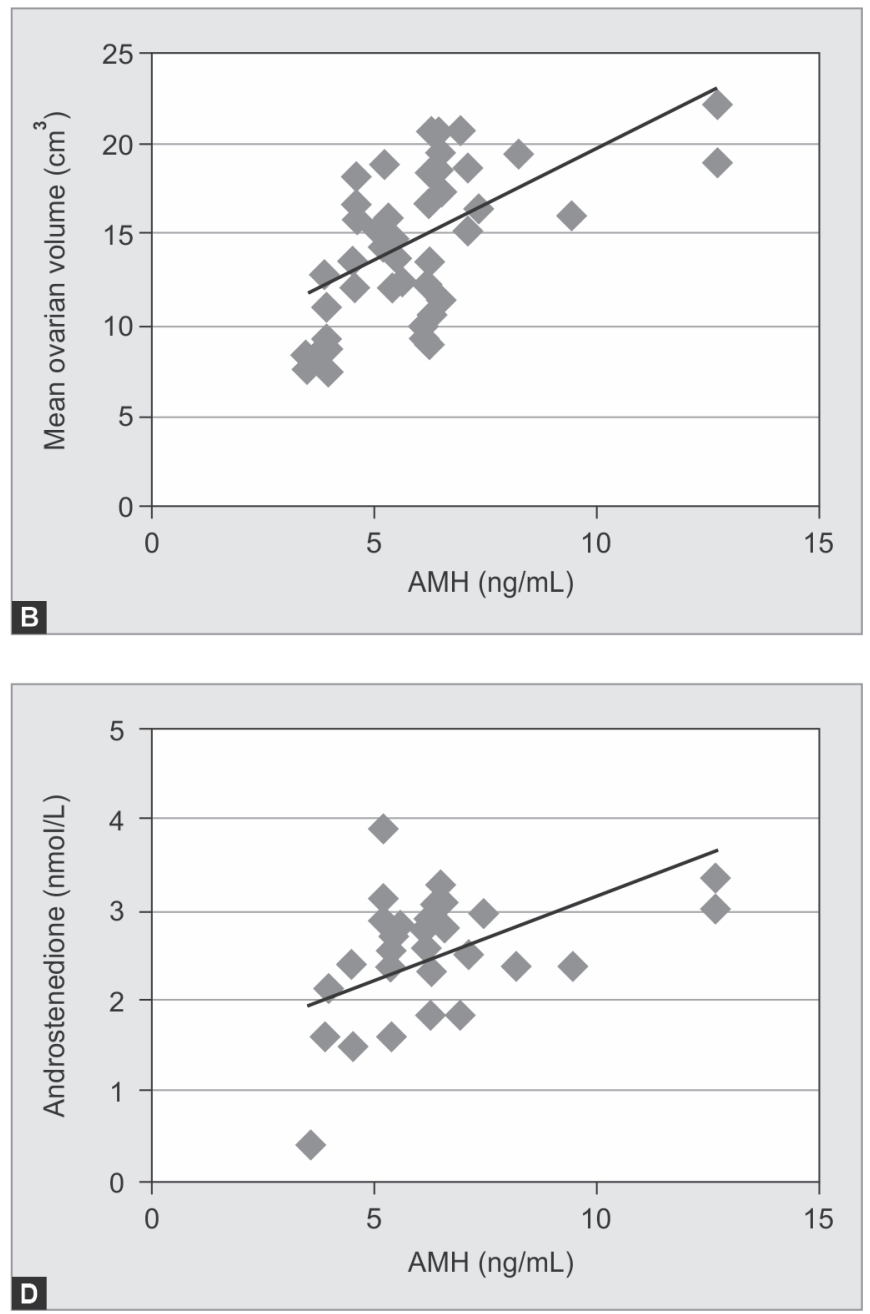

Figs $1 \mathrm{~A}$ to D: Correlation of serum AMH levels with hormonal and sonographic features in cases 

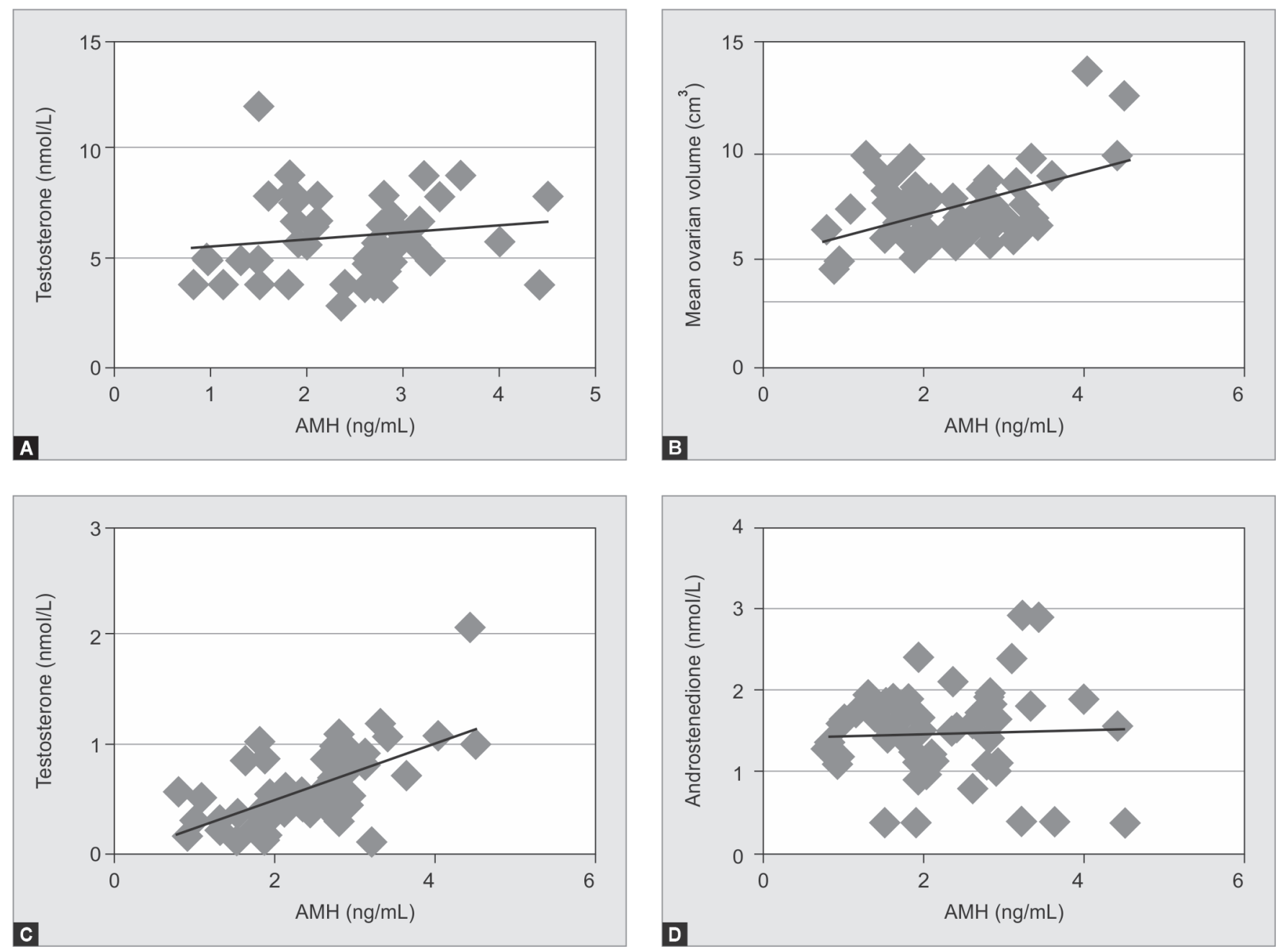

Figs 2A to D: Correlation of serum AMH levels with hormonal and sonographic features in controls

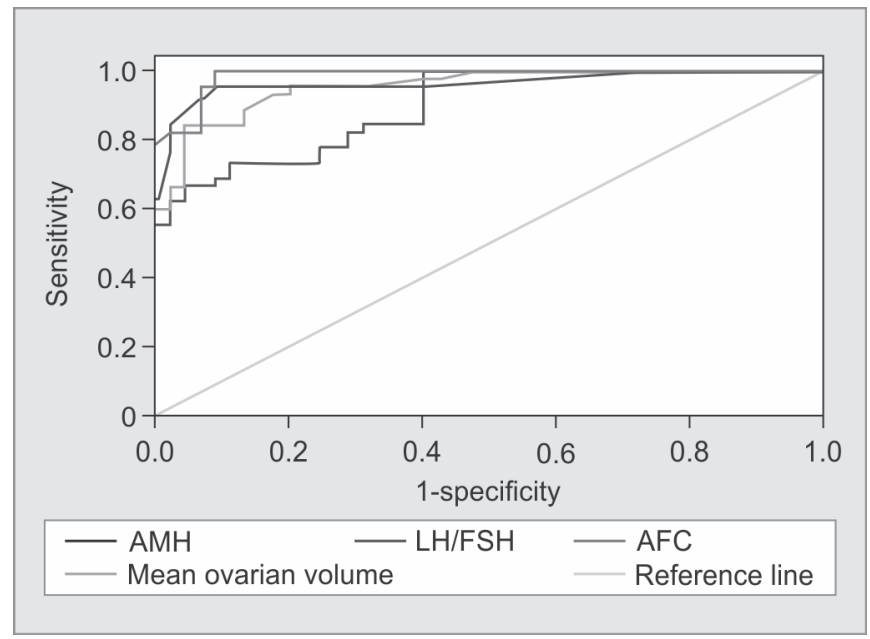

Fig. 3: Receiver-operating characteristic curve of $\mathrm{AMH}, \mathrm{LH} / \mathrm{FSH}, \mathrm{AFC}$ and mean ovarian volume

patients compared to controls, which is in agreement with other researchers. ${ }^{12-15}$

The AMH levels are reported to be higher in more severe disease phenotypes ${ }^{16}$ and correlate well with the individual features in the Rotterdam criteria. ${ }^{17,18}$
In our study, a significant negative correlation was seen between age and AMH levels in PCOS women. No such relationship was observed in controls. Laven et al. have also reported a significant negative correlation between age and $\mathrm{AMH}$ levels in both WHO Class 2 Infertile women and in controls. The decline with increasing age was more pronounced in PCOS women. ${ }^{7}$ Conversely, others have reported that PCOS blunts age-related decline in $\mathrm{AMH}$ levels. ${ }^{19}$ The mean age of PCOS women and controls in our study group was $24.1 \pm 4.7$ years and $25.2 \pm 4.6$ years, respectively. Since our study group and controls constituted primarily of relatively young women who are removed from the age of natural ovarian decline, we cannot conclusively comment on the impact of age on AMH levels in PCOS women.

It is observed that androgens promote the accumulation of small follicles and granulosa cells, thereby increasing AMH production. ${ }^{7}$ Wiweko et al. have observed that $\mathrm{AMH}$ values correlate with rising androgen levels and are highest for the phenotype exhibiting all three Rotterdam criteria. AMH also had a higher strength of association with $\mathrm{PCOS}(\mathrm{OR}=6.80)$ compared to the serum $\mathrm{LH}$ and FSH level (OR $=5.90$ and 4.90 respectively). ${ }^{15}$ In the present study, $\mathrm{AMH}$ levels correlated well with serum testosterone and androstenedione levels in PCOS women. A significant positive correlation was seen only with testosterone but not with androstenedione in controls. Similarly, in another study on women undergoing controlled ovarian 
Table 3: Threshold serum AMH levels for detection of PCOS in various studies

\begin{tabular}{|c|c|c|c|c|c|}
\hline Authors & Type of study & Number of participants & Threshold AMH & Sensitivity (\%) & Specificity (\%) \\
\hline \multirow[t]{2}{*}{ Saxena et al. ${ }^{12}$} & Prospective case-control & Cases 45 & $3.44 \mathrm{ng} / \mathrm{mL}$ & 77.78 & 68.89 \\
\hline & & Controls 45 & & & \\
\hline \multirow[t]{2}{*}{ Sahmay et al. ${ }^{13}$} & Cross-sectional & PCOS 419 & $3.94 \mathrm{ng} / \mathrm{mL}$ & 89.8 & 80 \\
\hline & & Controls 151 & & & \\
\hline \multirow[t]{2}{*}{ Zadehmodarres et al. ${ }^{14}$} & Cross-sectional & PCOS 60 & $3.15 \mathrm{ng} / \mathrm{mL}$ & 70.73 & 77.36 \\
\hline & & Controls 57 & & & \\
\hline \multirow[t]{2}{*}{ Wiweko et al. ${ }^{15}$} & Case-control & Cases 71 & $4.45 \mathrm{ng} / \mathrm{mL}$ & 76.1 & 74.6 \\
\hline & & Controls 71 & & & \\
\hline \multirow[t]{3}{*}{ Dewailly $^{25}$} & Prospective case-control & Non PCOS 105 & $5 \mathrm{ng} / \mathrm{mL}$ & 92 & 97 \\
\hline & & PCOS with HA/OA 73 & & & \\
\hline & & PCOS with $\mathrm{HA}+\mathrm{OA} 62$ & & & \\
\hline \multirow[t]{2}{*}{ Present study } & Cross-sectional & Cases 45 & $3.98 \mathrm{ng} / \mathrm{mL}$ & 82.2 & 93.3 \\
\hline & & Controls 45 & & & \\
\hline
\end{tabular}

stimulation, higher $\mathrm{AMH}$ levels were reported than in controls and the increase was greater among women with hyperandrogenism. ${ }^{20}$ Serum AMH levels also correlated with serum LH $(r=0.003, p=$ $0.0001)$ and mean testosterone levels $(r=0.477, p=0.0001)$ in WHO class 2 infertile women compared to matched controls. ${ }^{7}$ In 2,241 women of reproductive age with hyperandrogenic features with 17 hydroxy progesterone $>2 \mathrm{ng} / \mathrm{mL}$, serum AMH was significantly higher in those with PCOS than those with a late-onset congenital adrenal hyperplasia. They had suggested that serum AMH might be useful as a marker for PCOS in hyperandrogenic patients. ${ }^{21}$ Other researchers have also pointed to a positive correlation between androgens and AMH levels. ${ }^{12,22}$

$\mathrm{AMH}$ has been suggested as a substitute for sonographic findings especially where imaging is unavailable or is likely to be sub-optimal as in obesity and virginal status. Our data show a strong positive correlation of $\mathrm{AMH}$ levels with the mean ovarian volume and antral follicle count in PCOS women and with the mean ovarian volume alone in controls. Eilertsen et al. have reported that $\mathrm{AMH}$ levels are a good substitute for PCO morphology in the diagnosis of PCOS by both AES and Rotterdam criteria. ${ }^{23}$ Other researchers have also demonstrated a positive correlation of $\mathrm{AMH}$ levels with ovarian volume and antral follicle count. ${ }^{7,24,25}$

In our study, AMH was found to have good diagnostic potential for PCOS even as a stand-alone marker. It is a better predictor of PCOS than other parameters such as LH/FSH ratio, AFC and mean ovarian volume. Sahmay et al. have also suggested that AMH could serve as an important diagnostic marker in PCOS women with low or normal serum LH levels or normal LH/FSH ratio. ${ }^{13}$ They found that at a cut off of $3.8 \mathrm{ng} / \mathrm{mL}$ AMH had $81.6 \%$ sensitivity and $85.1 \%$ specificity for diagnosis of PCOS. Multiple authors have reported moderate to good sensitivity and specificity in predicting PCOS at levels ranging from 3.44 to $5.0 \mathrm{ng} / \mathrm{mL}^{12-15,25}$ (Table 3). However, a consensus on a single cut-off value remains elusive.

Researchers are examining the role of $\mathrm{AMH}$ as an adjunct and also as a replacement of the traditional PCOS criteria to improve the diagnosis of this disorder. ${ }^{12,13}$ It is therefore poised to emerge as an objective, reliable marker for disease severity and associated hormonal dysfunction in PCOS.

\section{Conclusion}

Polycystic ovarian syndrome is associated with higher AMH levels than controls. At a cutoff value of $3.98 \mathrm{ng} / \mathrm{mL}$, AMH has a sensitivity of $82.2 \%$ and specificity of $93.3 \%$ for diagnosis of PCOS. Its ability to predict PCOS is greater than that of AFC, mean ovarian volume, and LH/FSH levels. It correlates well with hyperandrogenemia and sonographic features of PCOS and could be used as a marker for androgen dysfunction and disease severity.

\section{References}

1. March WA, Moore VM, et al. The prevalence of polycystic ovary syndrome in a community sample assessed under contrasting diagnostic criteria. Hum Reprod 2010;25:544-551. DOI: 10.1093/ humrep/dep399.

2. Franks S. Polycystic ovary syndrome. N Engl J Med 1995;333:853-861. DOI: 10.1056/NEJM199509283331307.

3. Dumesic DA, Oberfield SE, et al. Scientific Statement on the Diagnostic Criteria, Epidemiology, Pathophysiology, and Molecular Genetics of Polycystic Ovary Syndrome. Endocr Rev 2015;36(5):487-525. DOI: 10.1210/er.2015-1018.

4. Dewailly $D$, Lujan ME, et al. Definition and significance of polycystic ovarian morphology: a task force report from the Androgen Excess and Polycystic Ovary Syndrome Society. Hum Reprod Update 2014 May-Jun;20(3):334-352. DOI: 10.1093/humupd/ dmt061.

5. Pepinsky RB, Sincalir LK, et al. Proteolytic processing of Müllerian inhibiting substance produces a transforming growth factor beta like fragment. J Biol Chem 1988;263:18961-18964.

6. Weenen C, Laven JS, et al. Anti-Müllerian hormone expression pattern in the human ovary: potential implications for initial and cyclic follicle recruitment. Mol Hum Reprod 2004;10:77-83. DOI: 10.1093/molehr/ gah015.

7. Laven JS, Mulders AG, et al. Anti-Müllerian hormone serum concentrations in normoovulatory and anovulatory women of reproductive age. J Clin Endocrinol Metab 2004 Jan;89(1):318-323. DOI: 10.1210/jc.2003-030932.

8. Cassar S, Teede HJ, et al. Polycystic ovary syndrome and antiMüllerian hormone: role of insulin resistance, androgens, obesity and gonadotrophins. Clin Endocrinol 2014;81:899-906. DOI: 10.1111/ cen. 12557.

9. Rotterdam ESHRE/ASRM-Sponsored PCOS Consensus Workshop Group. Revised 2003 consensus on diagnostic criteria and long-term health risks related to polycystic ovary syndrome. Fertil Steril 2004 Jan;81(1):19-25. DOI: 10.1016/j.fertnstert.2003.10.004.

10. Bhide $P$, Dilgil $M$, et al. Each small antral follicle in ovaries of women with polycystic ovary syndrome produces more antimüllerian hormone than its counterpart in a normal ovary: an observational cross-sectional study. Fertil Steril 2015 Feb;103(2):537-541. DOI: 10.1016/j.fertnstert.2014.10.033. 
11. Pellatt $L$, Hanna $L$, et al. Granulosa cell production of anti-Müllerian hormone is increased in polycystic ovaries. J Clin Endocrinol Metab 2007 Jan;92(1):240-245. DOI: 10.1210/jc.2006-1582.

12. Saxena U, Ramani M, et al. Role of AMH as Diagnostic Tool for Polycystic Ovarian Syndrome. J Obstet Gynaecol India 2018 Apr;68(2):117-122. DOI: 10.1007/s13224-017-1066-4.

13. Sahmay S, Aydin Y, et al. Diagnosis of Polycystic Ovary Syndrome: AMH in combination with clinical symptoms. J Assist Reprod Genet 2014 Feb;31(2):213-220. DOI: 10.1007/s10815-013-0149-0.

14. Zadehmodarres S, Heidar Z, et al. Anti-Müllerian hormon level and polycystic ovarian syndrome diagnosis. Iran J Reprod Med 2015;13(4):227-230

15. Wiweko B, Maidarti M, et al. Anti-Müllerian hormone as a diagnostic and prognostic tool for PCOS patients. J Assist Reprod and Genet 2014;31(10):1311-1316. DOI: 10.1007/s10815-014-0300-6.

16. Köninger $A$, Koch $L$, et al. Anti-Müllerian Hormone: an indicator for the severity of polycystic ovarian syndrome. Arch Gynecol Obstet 2014;290(5):1023-1030. DOI: 10.1007/s00404-014-3317-2.

17. Hayes $E$, Kushnir V, et al. Intra-cellular mechanism of Anti-Müllerian hormone $(\mathrm{AMH})$ in regulation of follicular development. Mol Cell Endocrinol 2016;433:56-65. DOI: 10.1016/j.mce.2016.05.019.

18. Seifer DB, Merhi Z. Is AMH a regulator of follicular atresia? J Assist Reprod Genet 2014;31(11):1403-1407. DOI: 10.1007/s10815-014-0328-7.

19. Piltonen $T$, Morin-Papunen $L$, et al. Serum anti-Müllerian hormone levels remain high until late reproductive age and decrease during metformin therapy in women with polycystic ovary syndrome. Hum Reprod 2005;20(7):1820-1826. DOI: 10.1093/humrep/deh850.

20. Eldar-Geva T, Margalioth EJ, et al. Serum anti-Müllerian hormone levels during controlled ovarian hyperstimulation in women with polycystic ovaries with and without hyperandrogenism. Hum Reprod 2005;20(7):1814-1819. DOI: 10.1093/humrep/deh873.

21. Tuten A, Sahmay S, et al. Serum AMH levels in the differential diagnosis of hyperandrogenemic conditions. Eur J Obstet Gynecol Reprod Biol 2014;177:121-125. DOI: 10.1016/j.ejogrb.2014.03.016.

22. Rosenfield RL, Wroblewski K, et al. Antimüllerian hormone levels are independently related to ovarian hyperandrogenism and polycystic ovaries. Fertil Steril 2012 Jul;98(1):242-249. DOI: 10.1016/ j.fertnstert.2012.03.059.

23. Eilertsen TB, Vanky E, et al. Anti-Müllerian hormone in the diagnosis of polycystic ovary syndrome: can morphologic description be replaced? Hum Reprod 2012 Aug;27(8):2494-2502. DOI: 10.1093/ humrep/des213.

24. Pigny $P$, Merlen E, et al. Elevated serum level of anti-Müllerian hormone in patients with polycystic ovary syndrome: relationship to the ovarian follicle excess and to the follicular arrest. J Clin Endocrinol Metab 2003 Dec;88(12):5957-5962. DOI: 10.1210/jc.2003030727.

25. Dewailly D. Diagnostic criteria for PCOS: Is there a need for a rethink? Best Pract Res Clin Obstet Gynaecol 2016 Nov;37:5-11. DOI: 10.1016/ j.bpobgyn.2016.03.009. 